\title{
HIGHLIGHTS
}

SCREENING

\section{Cell-bound proteins complement current screening options in patients with SLE}

Recent evidence suggests that measurement of the erythrocyte-bound complement activation products $\mathrm{C} 3 \mathrm{~d}$ (E-C3d) and E-C4d in patients with systemic lupus erythematosus (SLE) is more informative of disease activity than monitoring serum $\mathrm{C} 3$ and $\mathrm{C} 4$ levels. This finding, by Amy Kao and colleagues, might improve clinical care by providing a more accurate technique to track response to therapy in patients with SLE. "There is an unmet need for better biomarkers to diagnose and monitor disease activity in SLE," explains Kao, "so we examined whether changes in cell-bound complement levels reflected changes in disease activity and whether these new biomarkers added value to the traditional serum C3, C4 and anti-dsDNA antibodies as disease-monitoring tools."

The researchers measured E-C3d and E-C4d levels by flow cytometry in 157 patients with SLE, 290 individuals with other diseases (including Sjögren's
4 ...in patients with SLE ... E-C4d levels, but not serum C4 levels, were associated with disease activity... 7

syndrome and cutaneous lupus), and 256 healthy participants. At baseline, individuals with SLE had considerably higher E-C3d and E-C4d levels than those with other diseases and healthy controls. Furthermore, the investigators noted that in patients with SLE, E-C3d and E-C4d levels were highest in individuals classified as being 'more active' or 'most active' on the basis of refined Safety of Estrogens in Lupus Erythematosus: National Assessment (SELENA)-SLE Disease Activity Index (SLEDAI) or Systemic Lupus Activity Measure (SLAM) scores. By contrast, no correlations were observed between disease activity and serum C3 and C4 levels.
Kao's team next tracked disease activity over time in patients with SLE and found that E-C4d levels, but not serum C4 levels, were associated with disease activity, regardless of whether SELENA-SLEDAI or SLAM scores were used-both E-C3d and serum C3 levels showed varying degrees of correlation with disease activity.

SLE is a highly heterogeneous disease; however, the investigators envisage that screening E-C3d and E-C4d levels will advance clinical care in SLE by contributing to several distinct areas of endeavor. "Further investigation of E-C3d and E-C4d levels should be considered for potential use in routine care, clinical research, and testing of potential new therapeutic agents," conclude the researchers.

Rowan Higgs

Original article Kao, A. H. et al. Erythrocyte C3d and C4d for monitoring disease activity in systemic lupus erythematosus. Arthritis Rheum. 62, 837-844 (2010) 